\title{
Erratum
}

\section{A robust theory of resource allocation}

\section{Graciela Chichilnisky}

405 Low Library, New York, NY 10027, USA

In: Soc Choice Welfare (1996) 13:1-10

On page 3 in footnote 4 of the above paper the word "compactness" should be "convexity". The sentence should read as follows: "Convexity is not needed: all that is required is that the space $X$ be contractible, provided it is a locally convex space."

\section{Note to the reader}

The above paper by G. Chichilnisky is a response to the paper by B. Allen "A remark on social choice problem" published in the same issue (13:11-16) and should have been published after the paper by B. Allen. 